\title{
Task Partitioning: An Innovation Process Variable
}

\author{
Eric von Hippel \\ MIT Sloan School of Management \\ Cambridge, Mass. USA \\ (Published in Research Policy (1990) 19, 407-418.)
}

\begin{abstract}
Innovation projects are "partitioned" into smaller tasks. Precisely where the boundaries between such tasks are placed can affect project outcome and the efficiency of task performance due to associated changes in the problem-solving interdependence among tasks.

I propose that problem-solving interdependence among tasks can be predicted in many projects, and can then be managed by strategies involving (1) adjustment of task specifications, and/or (2) reduction of the barriers to problemsolving interaction across task boundaries.

The potential value of studying and managing task partitioning is illustrated by exploring how problematic areas of the innovation process, such as the designbuild and marketing-R\&D interfaces, can be better understood through that lens.
\end{abstract}

\section{Task Partitioning: An Innovation Process Variable}

\section{0: Introduction}

Innovation process researchers have studied how one might organize innovation most efficiently and effectively from many points of view. Interestingly, however, an almost vanishingly small element of this literature deals 
with the proper organization of innovation work with respect to the requirements of problem-solving. I propose that this is an important gap, because the core function of many innovation projects and project tasks is precisely problemsolving and the generation of new information. In this paper, therefore, I explore the link between innovation project "task partitioning" (how an innovation project is divided into tasks) and the efficiency and effectiveness of innovation project work.

I first characterize task partitioning (section 2). Next, I discuss the impact of partitioning on the on innovation project efficiency (section 3) and consider how the partitioning process might be improved (section 4). I then illustrate the practical importance of task partitioning by considering how problematic areas of the innovation process, such as the design-build interface and the marketing-R\&D interface, can be better understood via the lens the partitioning variable provides (section 5). Finally, I offer suggestions for further research (section 6).

\section{0: Dividing An Innovation Project Into Tasks}

An innovation project of any magnitude is divided up ("partitioned") into a number of tasks and subtasks that may then be distributed among a number of individuals, and perhaps among a number of firms. Task partitioning need not be completed prior to the start of project work. Indeed, as we will see later, the division of project work into tasks may appropriately proceed as the project itself proceeds. Nonetheless, if we were able to look at a completely partitioned project, what we would see is all component tasks and task interfaces specified, implicitly or explicitly, so that all would fit and work together to form the total project when combined. Such specifications say in effect: "This is the nature of Task X. These are the inputs it will or can receive from other parts of the project; and these are the outputs it must provide to specified points in the project at specified times."

Note that the partitioning being focused on involves the innovation work itself rather than the product or process resulting from that work. For example a new product may physically consist of components A and B. But the innovation project tasks leading to the development of that product may have been partitioned according to different boundaries, and it is the latter we are concerned with here.

Small innovation projects may be partitioned into tens or hundreds of component tasks. Large projects may be divided into thousands or even tens of thousands of such tasks, woven into an intricate network of interrelationships. 
Figure 1: Schematic of Simple Project Task Network

Figure 1 shows a simple project task network in schematic form. Here, tasks (or groups of tasks) are shown as circles or nodes, and interconnecting lines show the nature of task interdependencies - that is, show that the outputs of some tasks are required as the inputs of others.

Task networks can be drawn at a number of levels of aggregation depending on one's purpose. For example, if one is planning to manage the start-up of an entire auto company, "develop car model X" may well be shown as a single activity in a complex task network. But managers responsible for that project only might well devote an entire network to related tasks - perhaps to the level of detail of "develop front left door of car model X". Then, those in charge of door development work might in turn develop a network consisting of tasks at the level of "develop door locking mechanism", and so forth.

There are many different ways to partition a given project. Thus, in the instance of the "develop car model X" activity mentioned above, project participants might decide to specify "develop left front door" as a task, or they might decide to specify "develop left side of car" as a task and then specify subsidiary tasks such as "develop windows", etc., in a way that never isolates development of the door itself as a separate task. In many firms, certain task partitionings may be so traditional as to seem fixed - e.g., car firm Y may "always" have had a door development group. Nonetheless, task partitioning is in fact a manageable innovation process variable. 


\section{0: Task Changes, Problem-Solving Interdependence and Efficiency}

Project managers specify tasks and their interrelationships so that they can distribute innovation effort across people and organizations, both in parallel and in series with respect to time. It is the assumption that task boundary (input and output) specifications will be relatively stable as a project proceeds that allows project participants to focus on their own tasks, with some assurance that their output will properly mesh with the output of others to comprise the total intended project output.

Yet changes to task specifications and interrelationships are often required during the course of a project because planning errors were made, or because new information is introduced that was not available at that project's start. In the instance of innovation projects, changes for the latter reason are especially likely, because the core function of many innovation project tasks is precisely problemsolving and the generation of new information.

Changes caused by a single new information "event" may be restricted to only one task, or may affect the task network more widely. For example, suppose that a task in an auto development project involves research directed towards developing a very fuel-efficient engine. And suppose that research reveals that the originally planned approach to that problem will not succeed. Then, one might devise a new approach to achieving that same goal, in which case no other task need be affected by the new information. Or, one might decide that one will change other tasks or their interrelationships to compensate for the shortfall and maintain overall project performance. (For example, one might decide to try to lower friction in the drive train, and/or try to decrease the weight of the car to meet the original goal for fuel efficiency.) In this latter case the response of project participants to the new information clearly involves more extensive changes in the established task network.

With this example in mind, let me define the interdependence between any two innovation project tasks with respect to problem-solving as the probability that efforts to perform one of the tasks to specification will require related problem-solving in the other. The higher this probability in a given instance, the greater the problem-solving interdependence.

Changes introduced to task specifications after task work is under way can be costly because they often make what is already done valueless, and/or may 
degrade the solution ultimately arrived at, as project participants strive to "save" work already done by making suboptimal adaptations to change.

I propose that the cost of such changes will be less, other things being equal, if tasks are arranged so as to reduce the problem-solving interdependence among them. (Note that such partitioning changes will not necessarily reduce - or increase - the amount of problem-solving required in a given innovation project. They simply affect how that problem-solving is distributed among tasks.)

This proposal is not novel. In 1964, Christopher Alexander, an architect, proposed that the overall designs of houses or communities could be improved if they were made up of subsystems that could be adjusted relatively independently. Traditional designs had this characteristic, he said. He then argued that modern designers must strive to specify subsystems in their projects so that they were independent in this sense, lest the design problems they face become so complex as to be insoluble. (1)

In 1973 Herbert Simon made a similar argument with respect to "decisionmaking" tasks as follows:

"The division of labor is quite as important in organizing decisionmaking as in organizing production, but what is being divided is different in the two cases. From the information-processing point of view, division of labor means factoring the total system of decisions that need to be made into relatively independent subsystems, each one of which can be designed with only minimal concern for its interactions with the others. The division is necessary because the processors that are available to organizations, whether humans or computers, are very limited in their processing capacity in comparison with the magnitude of the decision problems that organizations face. The number of alternatives that can be considered, the intricacy of the chains of consequences that can be traced -- all these are severely restricted by the limited capacities of the available processors."

The information-processing rationale behind this criterion for problemsolving efficiency was noted by both authors, and is stated compactly by Simon in the paragraph quoted above. An argument for the criterion can also be made on organizational grounds as follows. Problem-solving that extends beyond a single individual involves communication and coordination among problem-solvers. A task boundary between problem-solvers often has associated with it physical and organizational barriers. Such barriers can add to the cost of problem-solvers' 
efforts to achieve cross-boundary communication and coordination, and thus reduce problem-solving efficiency. (Allen has shown that members of R\&D laboratories separated by either physical distance or or organizational barriers (e.g., membership in different groups) communicate far less frequently than do members not so separated.(3) )

The reader may gain a better intuitive feeling for the potential strength of the link between innovation project efficiency and the problem-solving interdependence of innovation tasks by considering two very simple and schematic examples. Each specifies an innovation project and then suggests two alternative ways to divide the project into two component tasks. These alternatives differ with respect to problem-solving interdependence between tasks and - as a consequence I suggest - also appear to differ with respect to the efficiency with which they can be carried out.

First, consider how one might partition the project of designing an airplane. In fact, of course, such a project would be partitioned into thousands of tasks. But, for present purposes let us assume that it will be partitioned into only two tasks, each to be undertaken by a different firm. The two alternative partitionings I propose we compare:

- "Firm $\mathrm{X}$ is responsible for the design of the aircraft body and firm $\mathrm{Y}$ is responsible for the design of the engine, and:

- "Firm $\mathrm{X}$ is responsible for designing the front half of the aircraft body and engine, and firm $\mathrm{Y}$ is responsible for the back half of each.

Taken together, each of these proposed partitionings has the same project outcome - a complete aircraft design. But the two differ greatly with respect to the interdependence of the two tasks specified. The second alternative would require a much higher level of problem-solving between the two tasks. For example, many design decisions affecting the shape of the "front half" of an aircraft body could not be made without forcing related changes on the designers of the back of the body and vice versa: The two halves cannot be considered independently with respect to aerodynamics. In contrast, the detailed design of a complete aircraft engine is much less dependent on the detailed design of a complete aircraft body. As a direct consequence, I suggest, engineers would think the former partitioning far more efficient than the latter. Indeed, faced with the latter proposed division, 
experts would be likely to throw up their hands and say, "It can't be done that way".

As a second example, consider how one might partition the project of designing the interiors of two rooms between two interior decorators. One might assign each room to each decorator; one might assign one-half of each room to each. Again, the same work is to be accomplished in each instance. Only the way it is divided up has been changed.

In this example, the idea of two interior decorators each designing one-half of a room probably seems absurdly inefficient to the reader. And again, I propose that this is because of the need for between-task problem-solving that is inferred. That is, it seems reasonable that a solution devised by one decorator and implemented on one side of a room must cause the second artist to make responsive adaptations on the other side of the room if a satisfactory total design is to result.

We can see that it is the need for problem-solving across tasks that makes these partitionings seem inefficient by slightly changing the nature of the task in this second example. Suppose that problem-solving is clearly not involved in the room-design project. For example, suppose that the physical task is the same two interior decorators are each assigned one-half of a room to design - but suppose that the decorators work for a hotel chain and proceed according to a strict formula. In that case, asking each decorator to design half a room might be a perfectly acceptable, and possibly even efficient, partitioning of the task. For example, one decorator could specialize in applying the formula to window decorations, and one could specialize in applying it to room furnishings.

\section{0: Understanding and Managing Task Partitioning}

If improvements to innovation task partitioning can yield major benefits to firms, we then have much to learn about it. Firms I have interviewed to date appear not to think about task problem-solving interdependence as an input to partitioning decisions at all, at least not explicitly. Instead, some appear to make such decisions primarily on the basis of assumed economies of specialization (e.g.: "All electrical design work will be done by group A"; "All marketing research studies will be done by group M"). Other firms appear to simply follow some traditional pattern of innovation task partitioning without analysis. (E.g.: "We have always designed aircraft bodies by dividing them into a series of cylindrical 
sections and assigning each section to a different task group. No one now at the company has thought about why we do this or whether it currently makes sense from any point of view. It is just the way we do it.")

As a start towards research on the matter, let me consider two complementary approaches to managing the problem-solving interdependence of innovation project tasks. These are: (1) specification of tasks so as to reduce the need for problem-solving across task boundaries; (2) reducing the cost of engaging in a given level of problem-solving across task boundaries.

\section{Specification of Tasks}

In order to specify tasks so as to reduce problem-solving interdependence among tasks we must be able to, first, predict which tasks are likely to be the source of important new information; second, predict which other tasks in the network are likely to be affected by that new information; third, use such predictions to adjust task specifications. I propose that these things can be done to a useful degree both in the instance of "routine" innovation projects, and in the instance of "very novel" ones. Let me consider each of these project types in turn.

Most innovation projects in most firms do not involve great novelty. Thus, computer companies specialize in repeatedly developing the next new model computer, and auto firms specialize in repeatedly developing the next new auto model. Under such conditions, innovators learn much from prior projects as to areas where change is likely to be needed during the course of a future, similar innovation project. For example, an engineer experienced in auto design work can look at specifications for a new model car and easily predict the areas where the most difficult design problems are likely to be encountered during the course of the project.

The way that changes to a given task are likely to propagate across a task network can also often be predicted by project personnel who have experience with similar projects. Thus, changes in the design of some components of an auto engine can be expected, with a near-certainty, to require changes in other design tasks, because the tasks are predictably interdependent with respect to problemsolving. For example, problem-solving with respect to the shape of the top of a piston in an auto engine is predictably very interdependent with the task of problem-solving with respect to the shape of a cavity in the cylinder head of an auto engine: The two shapes combine to form the overall shape of an auto engine combustion chamber, and the shape of the combustion chamber is an important 
variable in engine design. In contrast, the likelihood that changes to either or both of these components will require changes in the tasks of designing many other parts of the auto engine - ranging from alternator to engine mounts - will be seen as predictably very remote by those who understand auto engine design.

In sum, in the instance of routine innovation projects, it is reasonable to expect that project participants can predict which tasks are likely to be the source of important new information, and which other tasks in the network are likely to be affected by that new information. Therefore in the case of such projects, one can obtain the information needed to readjust task boundaries so as to minimize the need for cross-boundary problem-solving. Indeed under these conditions, one may be able to predict the course of problem-solving so well as to be able to lay out and partition many tasks at a very early stage in an innovation project.

In the instance of "very novel" projects, an ability to predict the source and pattern of problem-solving at the outset of that project is equivalent to saying that we be able to predict the unexpected - not a very promising prospect. However, one can still improve task partitioning with respect to problem-solving interdependence under these conditions by partitioning as the project unfolds.

Consider what we know about the nature of the engineering problemsolving process. Marples (4) and others have found that engineering problem definition and related problem-solving can evolve as a project progresses. Thus, one may start a project only knowing that one wants to build a computer that is "beyond the state of the art" with respect to speed at performing certain types of computation. But many designs may allow one to reach the specified goal, so the designer's first step is to generate and analyze alternative approaches in a preliminary way. (E.g.: "We could use a single superfast processor or use multiple processors in parallel".) As work proceeds, the different subproblems (tasks) that are associated with each approach become clearer. (For example, engineers may identify the key subproblems associated with the parallel processor approach as innovative computer architecture and software. In contrast, the key subproblems in the instance of a single processor approach may be determined to be the design and fabrication of a processor faster than any made before.) The team elects to follow the approach(es) it considers most promising further, and elects to abandon others on the basis of early findings. As any approach is followed further "sub-sub problems" specific to that particular approach emerge, and so forth.

Obviously one cannot partition a task before one knows what it is. But, in the scenario just described, it is quite possible to partition emerging tasks as the 
project unfolds. One simply thinks through what one can predict about the course and pattern of problem-solving as work progresses, and partitions as best one can with an eye towards minimizing task problem-solving interdependence. Such an effort is not very risky, because full accuracy in prediction regarding the source and pattern of problem-solving activity in a project is not required in order to gain from improved task partitioning. This is because all project tasks are not likely to be interdependent, and the effects of a bad partitioning choice made in one area of a project therefore will not necessarily propagate to affect the efficiency gains resulting from good choices made elsewhere.

Currently, I am not aware of any methods that can enhance practitioner insight with respect to identifying likely areas and patterns of change in an innovation project. Tools do exist, however, that can help managers to record and to think through the implications of their insights. Early efforts to devise and experiment with tools for this purpose $(5,6,7)$ were not picked up by practitioners insofar as I can determine. However, a graphical method called "QFD" has recently been developed by practitioners, and is apparently receiving considerable use.

QFD encourages the placing of customer requirements, engineering requirements and manufacturing requirements with respect to a proposed project onto a common matrix, so that interactions and possible conflicts can be identified and discussed by project team members at an early stage (8). Thus, the method might highlight the following interaction: "The better an auto door is at tightly sealing out noise and dirt (a desirable characteristic), the harder it is to close (an undesirable characteristic) given that conventional sealing technology is applied". Such information can be used as an aid to improving task partitioning. Thus, if project specifications require improvements in door closing or door sealing, the presence of the interaction with respect to these two matters suggests that arranging task partitions so that both are included in a single "improve door closing and door sealing" task would reduce task problem-solving interdependence in this instance.

\section{Ease Cross-Boundary Problem-Solving}

A second approach to managing task problem-solving interdependence involves reducing the cost of engaging in problem-solving across task boundaries. This approach is complementary to the one discussed above: It regards existing task partitions as given, and seeks ways to minimize the costs of any associated 
cross-boundary problem-solving. Therefore, both approaches can be applied simultaneously when attempting to manage the effects of task problem-solving interdependence.

There has long been a literature in the field of organizational behavior that explores ways to pass information across group boundaries, or to integrate groups divided by a boundary. Therefore some tools for lowering the cost of problemsolving across boundaries are reasonably well understood. Naturally occurring mechanisms to this end such as the "gatekeeper" who takes on the role of passing information between organizations have been described.(9) Also, various inventions developed specifically to accomplish boundary spanning such as the "integrating group" (10) have been described by specialists and been applied in the field.

One could address such tools to our present problem by first predicting where high interaction between tasks will be required, and/or monitoring task activities to identify such needs as they arise. Then, special efforts could be made to ease communication across the relevant task boundaries. Thus, in this second approach one might keep the door-closing and door-sealing activities described earlier as two independent tasks. But, upon noting the problem-solving interdependence between them, one could take steps to facilitate problem-solving interaction across that particular boundary.

There is also a more recent literature reporting on the experience of some Japanese firms in this matter. Here, the emphasis appears to be less on specific mechanisms intended to span a particular boundary for a particular end, and more on organizational designs that encourage a general high level of communication and a reduction of barriers to communication and joint problem-solving between all project tasks.

Thus, Imai, Nonaka and Takeuchi report that five very successful product development projects by Japanese firms used multifunctional development teams of 30 or fewer people to develop relatively complex products having a significant degree of novelty in their fields. (Honda, the 'City' car; Fuji-Xerox, the FX-3500 copier; Canon, the 'Sure Shot' camera; NEC, the PC 8000 personal computer; Epson, the MP-80 dot matrix computer printer). They point out many ways in which these teams were designed to maximize within-team information flow and minimize task boundaries, and contrast this with US product development practice. Thus, they note that project phases such as product concept, feasibility, definition and design - often sharply demarked in US practice with progress 
reviews and approvals - were much less defined and overlapped more heavily in the practice of the Japanese teams:

"The loose coupling of [project] phases also makes the division of labor, in the strict sense of the word, ineffective. Division of labor works well in a [US style] system where the tasks to be accomplished in each phase are clearly delineated and defined. Each project member knows his or her responsibility, seeks depth of knowledge in a specialized area, and is evaluated on an individual basis. But such segmentalism ... works against the grain of a loosely coupled system [such as that observed in the Japanese development projects]. Here, the norm is to reach out across functional boundaries as well as across different phases. Project members are expected to interact with each other extensively, to share everything from risk, responsibility [and] information to decision-making, and to acquire breadth of knowledge and skills."(11)

Under such conditions, barriers between many project tasks may indeed be very low and flexible, thus reducing the need to make special accommodation for those tasks having high interdependence with respect to problem-solving.

\section{0: Practical Importance}

Is management of the problem-solving interdependence of tasks a matter of any practical importance to innovators? I think so, and will illustrate the possibility anecdotally by looking at two areas in the innovation process that are known to be problematic through the lens of task partitioning. The first of these is commonly called the "marketing-R\&D" or "marketing research - product design" interface in the innovation process literature. The second is typically referred to as the "product design - process design" or "design-build" interface in that literature. These two interfaces are the boundaries between the three tasks of marketing research, product design and process design. All three tasks are typically carried out (in series or with some time overlap) during the course of an innovation project. In what follows, I will first consider the "design-build" interface in some detail. Then I will more briefly incorporate the marketing-R\&D interface into the discussion.

\section{The Product Design - Process Design Interface}


Traditionally, the interface between product design and process design has been a source of difficulty in the progress of an innovation project. Traditionally, also, this difficulty has been framed as the problem of transferring information smoothly and completely from the former to the latter.(12) More recently, however, the underlying assumption of a one-way flow from product design to process design has begun to be seen as a problem in itself. In essence, researchers and practitioners now better appreciate that the product and process design tasks can interact in a way that requires two-way communication and joint problemsolving between the groups engaged in these. That is, the way you design a product has implications for process design - and vice versa.

For example, a product designer may initially design a component part that is very difficult to manufacture - despite instructions and good intentions - because he does not understand manufacturing processes well. He will typically be able to change his design to resolve the problem - if told of the difficulty in a timely manner. But if product designers only provide information to process designers at the completion of product design, there will obviously be no opportunity for process designers to provide the needed data during the time when the design work is still under way and changes could be relatively easily accommodated.

Two empirical studies I am aware of have examined the design-build interface, and have shown that an increase in interaction across this boundary is associated with increased project efficiency. Thus, authors of a study of the matter in the commercial, power, light industrial and heavy industrial segments of the construction industry found that:

"a construction specialist [building constructor], working with the engineering team [building designers] as the project is defined and designed, can cut costs by 10 to 20 times the added cost of extra personnel. On a $\$ 30$ million project, an extensive constructability program may cost $\$ 50,000$, but can bring savings of $\$ 1$ million. Costs and schedules are trimmed by:

- Arranging the optimum preparation of both engineering details and the sequence in which they are prepared so as to avoid delays in construction on the site.

- Taking advantage of the latest construction technology as part of the design. 
- Developing work-simplifying methods and minimizing labor-intensive design."(13)

Similarly, Clark et al. (14), in a recent comparison of aspects of the European, Japanese and US auto industries, provide a detailed case study of how information was passed between designers of the sheet-metal parts that make up the surface of an automobile, and the designers of the dies used to produce these parts. In the Japanese firms, they found, the work of parts designers and dies designers had a larger overlap with respect to time than did US and European firms. They also found that Japanese parts designers typically passed preliminary information more frequently to die designers regarding the planned shape of parts as work progressed.(15) As a consequence, they suggest, die designers in the Japanese firms were in a better position to begin the design of dies while some areas of shape were still uncertain, and to suggest changes to part designers in a timely fashion that would reduce the cost, complexity or number of dies required to make the part.

On the basis of studies such as these, plus anecdotal reports of good results in various firms with "design-build" teams, practitioners and researchers are now moving to the view that closer interaction between product and process design is in general beneficial. However, if we view this problem through the lens developed in this paper - that one wants to partition tasks so as to minimize the need for problem-solving across task boundaries and/or build bridges between tasks anticipated to require high problem-solving interaction - then we can advance the discussion a step further, in my view.

I propose that the level of benefit obtained from bridging or eliminating the task boundary between product design and process design will differ as a function of the particular part and process at issue. This is because, as discussed earlier, the need for problem-solving across such a boundary can vary depending on the particular part and process involved, and depending upon the specifications set for task outcomes.

Thus, in the case study by Clark et al. mentioned above, the design of auto parts and of the dies used to produce them are clearly very interdependent design tasks, and it therefore seems reasonable that the bridging or elimination of the boundary between these two tasks would improve innovation process efficiency and effectiveness. 
On the other hand, I would suspect that one would not typically get a similar benefit by bridging or eliminating the task barrier between product design and process design in the instance of "middle-of-the-road" printed circuits. This is because standard printed circuit manufacturing technology is capable of producing any such ordinary circuit without any process adjustments being needed or useful. That is, there will not usually be a need for joint problem-solving - or two-way communication - between these two tasks. (Those unfamiliar with printed circuit technology can think of book printing as a useful substitute example. Book authors typically do not have to write their books with the needs of the printer in mind, because the ordinary printing process does not have to be adapted to the particular words chosen by an author. In contrast, a graphics designer trying to do something that pushes the limits of existing printing processes might well find joint problem-solving with the printer to be very valuable.)

The need to make choices with respect to where one will eliminate task boundaries and/or increase interaction across them can be illustrated by reference to the practice of some auto manufacturers and others of sometimes specifying components they want simply as "black boxes". In such cases the work of designing the components in detail is assigned to the supplier firms that will manufacture them.(16)

Figure 2A

Auto Firm: $\quad$ Design Component A Design Component B

Component B Build Component B

Manufacturer:

Figure 2B

Auto Firm: $\quad$ Design Component A 
Component B

Manufacturer:
Design Component B

\section{Build Component B}

Figure 2: Shifting the detailed design of one product component (Component B) from the firm designing the product to the firm that will manufacture it improves the design-build linkage for Component B - but weakens the design linkage between Component A and Component B.

As is suggested in Figure 2, a shift of the detailed design of automobile component $\mathrm{B}$ from the firm that designs the overall automobile to the firm that builds the component probably increases the barriers between the design of component $\mathrm{A}$ and component $\mathrm{B}$, while decreasing the barrier between product design and process design for these components. After all, the shift involves a shift in the physical and organizational location of the component design work from a close(r) proximity with other design tasks, and to a closer proximity with process design tasks. And, as mentioned earlier, Allen (3) has shown that both physical and organizational distance presents significant barriers to technical communication.

Clark et al. have found the assignment of greater amounts of detailed component design work to component suppliers to be strongly associated with a reduction in the time required to develop a new model car. Indeed, they estimate that "bringing an additional twenty percent of the engineering effort in-house and inside the project [that is, assigning less of the detailed component design work to the component supplier] would add eight months to project lead time."(17).

In line with arguments made earlier, I suggest that further examination will show that the effect of this shift is positive only for those components where there is less benefit obtainable from problem-solving interaction between particular component design tasks than between the tasks of product design and process design. For example, I suspect that shifting the detailed design of an electrical alternator (generator) to be used in a new model car from the auto design firm to the component manufacturer would result in a net improvement in efficiency: there is little design trade-off between generator design and the design of other components. 
On the other hand, if the component in question is the plastic ducting used to distribute hot and cold air to a car's interior, I would expect the reverse to be true. Such ducting is bulky, and must be laid out with an intimate knowledge of the location and size of many other auto components if it is to be fitted within the car properly. To carry out this design process efficiently, it seems to me, there would be a need for creative, interactive problem-solving involving the designers of the car as a whole and the designers of the air ducting components. Thus, the gain from reducing the barriers to such interaction by keeping both design tasks within the auto manufacturer seems to me likely to outweigh any advantage to be gained from shifting detailed design to the manufacturer in this instance.

\section{The Marketing Research - Product Design Interface}

The interface or boundary between the marketing-R\&D interface has long been judged to be a source of problems with respect to the commercial success of innovation projects because most new products developed fail in the marketplace (18); accurate understanding of user need has been shown key to innovation project success (19); engineers and marketers are often unhappy with the quality of the information regarding user need transmitted across the marketing-R\&D interface (20).

As was the case with the design-build interface, the "interface problem" has been traditionally seen as one of smooth and accurate transfer of "need" information from marketing to product designers. Today, however, improved twoway communication across this interface is now clearly identified as a likely way to improve performance at this interface.(21) However, as in the case of the design-build interface, I suggest that the benefit realizable from repartitioning tasks to eliminate the boundary between marketing research and product design, or taking steps to ease problem-solving across that boundary, will depend on the amount of joint problem-solving required across the interface in any particular instance.

Thus, a single one-way message from marketing to product design may suffice if the need information is unambiguous, and if the designers can accommodate the request without compromising other product characteristics. E.g., "The customer wants this software to interface with the XYZ printer." On the other hand, joint problem-solving between marketing researchers, customers, and product designers will clearly be valuable when, for example, data on new product needs provided by marketing research to engineering have consequences 
or offer opportunities that are not initially visible to all these parties. E.g., "Are you aware that adding memory to the computer as you request will make it slower?" Or, "Do you realize that if we add extra memory we can also add feature $\mathrm{X}$ at no cost?"

\section{0: Discussion and Suggestions for Further Research}

In this paper I have proposed that the level of problem-solving interdependence between tasks is a function of choices made with respect to task partitioning. I have also suggested that such choices can be managed, and that doing so may have an important impact on innovation process efficiency. It seems to me that these proposals are worth exploring further from the point of view of both innovation process research and innovation practice.

Innovation task partitioning is potentially a very interesting topic in the field of innovation process research because it bears directly on the matter of how innovation can be most efficiently distributed within and among firms. Findings related to partitioning can therefore serve as a useful input to research on topics ranging from specialization to vertical integration to the role of suppliers in the innovation process.

In such research, the conditional nature of improvements to innovation process efficiency as a result of changes in task partitioning will become evident and important. That is, decisions regarding partitioning influence far more than problem-solving efficiency. They also have an impact on matters ranging from the efficient utilization of specialized resources, to the ability of a firm to protect its innovation-related advantages in the marketplace. Sometimes partitioning from the point of view of minimizing problem-solving interdependence among tasks will have positive impacts on these related matters, but sometimes a trade-off of benefits will be required. (E.g., "We would gain efficiency if the manufacturer of component $\mathrm{X}$ worked with our designers at an early stage, but this strategy would also increase our risk that news of our new product might leak out early and reduce our profits.")

Since there is more than one solution possible to most design problems, the way a project is partitioned into tasks will also strongly influence the nature of the solution ultimately developed by a project team. Thus, an auto firm project team that chooses to design an auto engine and auto transmission as one task will probably come up with a different design - and will surely learn more about 
engine-transmission interactions - than a team that partitions engine design and transmission design into separate tasks.

An improved understanding of innovation task partitioning will be important to innovation managers if and as it can have an important impact on innovation project efficiency and effectiveness. The magnitude of this effect needs to be empirically explored. As an initial step, it might be reasonable to attempt to view the efficiency effects of innovation project task partitioning in isolation. One might be able to do this experimentally by, for example, systematically varying the way a given development project is divided into modules (tasks) and observing the effect of these variations on project performance and outcomes.

Next, it might be reasonable to explore the possibility of managing innovation task partitioning in practice to achieve efficiency and effectiveness gains in innovation project work. For example, one might select a sample of innovation projects for study, list the major tasks associated with each, and then ask project participants to: (1) rank the degree of problem-solving interaction required among different listed tasks, and (2) rank the ease of accomplishing such problem-solving among these same tasks. A large mismatch between the answers to the two questions might indicate that practically useful task partitioning changes could be made, and would also suggest where these might lie.

If work such as that described above does show that improvements in task partitioning with respect to problem-solving can offer major benefits to firms, we must learn more about how to manage it. In this regard, it is important to point out that "management" does not necessarily imply management from the top or by specialists: Indeed, it is very possible that decisions regarding task partitioning and needed communication across task boundaries can be most effectively managed at the working level. After all, that is where problem-solving is done and where the fast-changing data on the nature of needed problem-solving originate. (Interestingly, firms that try to manage task partitionings at a higher level may find project participants making informal and perhaps covert adjustments in any case (22).)

I look forward to studying innovation task partitioning further, and very much hope that others will also find the area interesting enough to explore. 


\section{References}

1. Christopher Alexander, Notes on the Synthesis of Form (Cambridge, Mass.: Harvard University Press, 1964), chapters 2-5.

2. Herbert A. Simon, Administrative Behavior: A Study of Decision-Making Processes in Administrative Organization, 3rd ed. (New York: Free Press, 1976), chapter 14, p. 293. This chapter was taken with minor revisions from a 1973 article, "Applying Information Technology to Organization Design," Public Administration Review 33 (1973): 268-78.

3. Thomas J. Allen, Managing the Flow of Technology (Cambridge, Mass.: MIT Press, 1977), chapters 7 and 8.

4. David L. Marples, "The Decisions of Engineering Design," IRE Transactions on Engineering Management, June 1961: 55-71.

5. C. Alexander and M. L. Manheim, "HIDECS 2: A Computer Program for the Hierarchical Decomposition of Systems which have an Associated Linear Graph," Research Report R 62-2 (Cambridge, Mass.: Civil Engineering Systems Laboratory, Massachusetts Institute of Technology, 1962); C. Alexander and M. L. Manheim, "HIDECS 3: Four Computer Programs for the Hierarchical Decomposition of Systems which have an Associated Linear Graph," Research Report R 63-27 (Cambridge, Mass.: Civil Engineering Systems Laboratory, Massachusetts Institute of Technology, 1963).

6. W. P. Lewis, A. E. Samuel, and B. W. Field, "An Example of the Application of a Systematic Method to Design," Operational Research Quarterly 24, no. 2 (1973): 217-33.

7. John Luckman, "An Approach to the Management of Design", Operational Research Quarterly 18, no. 4 (1967): 345-58.

8. John R. Hauser and Don Clausing, "The House of Quality," Harvard Business Review 88, no. 3 (May-June 1988): 68-72.

9. Thomas J. Allen, Managing the Flow of Technology (Cambridge, Mass.: MIT Press, 1977), 141.

10. Paul R. Lawrence and Jay W. Lorsch, "Organizing for Product Innovation," Harvard Business Review (January-February 1965): 109-120.

11. Ken-ichi Imai, Ikujiro Nonaka, and Hirotaka Takeuchi, "Managing the New Product Development Process: How Japanese Companies Learn and Unlearn," in The Uneasy Alliance, edited by K.B. Clark, R.H. Hayes, and C. Lorenz (Boston: Harvard Business School Press, 1985), $337-75$.

12. J.A. Morton, Organizing for Innovation: A Systems Approach to Technical Management (New York: McGraw-Hill, 1971). 
13. "Integrating Construction Resources and Technology Into Engineering," A Construction Industry Cost Effectiveness Project Report (New York: The Business Roundtable, August 1982).

14. Kim Clark, W. Bruce Chew, and Takahiro Fujimoto, "Product Development in the World Auto Industry: Strategy, Organization and Performance," paper presented at the Brookings Microeconomics Conference, 3-4 December 1987.

15. Ibid., 35-37.

16. Ibid., Table 1, p. 15.

17. Ibid., 23.

18. Glen L. Urban and John R. Hauser, Design and Marketing of New Products (Englewood Cliffs, N.J.: Prentice-Hall, 1980), 53-55.

19. R. Rothwell, C. Freeman, et al., "SAPPHO Updated -- Project

SAPPHO Phase II," Research Policy 3 (1974): 258-91.

20. Ashok K. Gupta and David Wilemon, "The Credibility-Cooperation Connection at the R\&DMarketing Interface," Journal of Product Innovation Management 5, no.1 (March 1988): 20-31.

21. William E. Souder, "Managing Relations Between R\&D and Marketing in New Product Development Projects," Journal of Product Innovation Management 5, no.1 (March 1988): 619.

22. Henry B. Eyring, "Some Sources of Uncertainty and Their Consequences in Engineering Design Projects", IEEE Transactions on Engineering Management, EM-13, no. 4 (December 1966): 167-80. 